\title{
The Effects on the Cognitive Function of Healthy Volunteers of a Combination of Acetyl-L-Carnitine, Vinpocetine and Huper- zine A Administered Over 28 Days
}

\author{
Keith A Wesnes ${ }^{1,2,3^{*}}$ and Josh Reynolds ${ }^{4}$ \\ ${ }^{1}$ Wesnes Cognition Ltd, Little Paddock, Streatley Hill, Streatley on Thames, UK \\ ${ }^{2}$ Visiting Professor, Department of Psychology, Northumbria University, Newcastle, UK \\ ${ }^{3}$ Adjunct Professor, Centre for Human Psychopharmacology, Swinburne University, Melbourne, Australia \\ ${ }^{4}$ MedHealth Direct, Laguna Beach, California, USA \\ *Corresponding author: Keith A Wesnes, Professor, Wesnes Cognition Ltd, Little Paddock, Streatley Hill, Streatley on \\ Thames, RG8 9RD, UK
}

\begin{abstract}
This report on a 4 week, randomized, placebo controlled, parallel group, repeated measures, double-blind study clinical trial of a preparation containing Acetyl-L-Carnitine, Vinpocetine and Huperzine $A$ administered to healthy volunteers has identified that the product significantly improves a well validated global score from six tests of working and episodic memory, with an effect sized improvement comparable to the current medications approved to treat Alzheimer's disease.
\end{abstract}

\section{Keywords}

Acetyl-L-Carnitine, Vinpocetine, Huperzine A, CDR system, Memory, Attention, Automated cognitive testing

\section{Introduction}

Procera $\mathrm{AVH}$ is a proprietary combination of the three widely studied compounds - Acetyl-L-Carnitine, Vinpocetine and Huperzine A. Preclinical work on each compound has established mechanistic bases which could enhance human cognitive function as well as treat cognitive impairment. Acetyl-L-Carnitine administered orally actively passes from the blood stream into the brain [1] where it exhibits a variety of beneficial effects. These include promoting cellular energy production, the removal of toxic accumulations of fatty acids $[2,3]$ and elevating levels of nerve growth factor $[4,5]$. Acetyl-L-Carnitine directly stimulates the cholinergic system
[6] and can be converted to acetylcholine [7]. Vinpocetine has been found to improve cerebral metabolism, increase glucose and oxygen consumption by the brain as well as to improve its resistance to hypoxia [8]. It also improves cerebral microcirculation and blood flow by inhibiting platelet aggregation [9], improving red blood cell deformability [10] and reducing cerebral vascular resistance. It also has neuroprotective effects through blocking voltage-gated sodium channels [11] and increasing the effects of adenosine [12]. Huperzine $A$ is an anticholinesterase inhibitor which exerts its effects via the brain cholinergic systems.

These compounds have been studied a variety of clinical trials in volunteers, aged individuals as well as patients with cognitive disorders associated with pathological ageing including Alzheimer's and other dementias. Acetyl-L-Carnitine has been the most widely studied of the compounds, and Montgomery, et al. [13] performed a meta-analysis of the 21 double-blind, placebo-controlled clinical trials in Mild Cognitive Impairment or Alzheimer's disease which were conducted for 3 months or longer. A statistically significant advantage for Acetyl-L-Carnitine over placebo was seen in the pooled effect size for cognitive improvement of 0.201 (95\% confidence intervals $0.107 \& 0.295$ ) over the 21 studies. Several studies have shown cognitive benefits on vinpocetine in patients with dementia [14-16]. Fur-

Citation: Wesnes KA, Reynolds J (2019) The Effects on the Cognitive Function of Healthy Volunteers of a Combination of Acetyl-L-Carnitine, Vinpocetine and Huperzine A Administered Over 28 Days. Int J Neurol Neurother 6:089. doi.org/10.23937/2378-3001/1410089 Accepted: September 12, 2019: Published: September 14, 2019

Copyright: (C) 2019 Wesnes KA, et al. This is an open-access article distributed under the terms of the Creative Commons Attribution License, which permits unrestricted use, distribution, and reproduction in any medium, provided the original author and source are credited. 
ther Huperzine A has shown beneficial effects in patients with dementia as well as in healthy volunteers in a scopolamine model of Alzheimer's disease [17].

Overall, there is sufficient clinical data to support a clinical trial to evaluate the prediction that a combination of these three products would favourably affect major domains of cognitive function in healthy volunteers. Preliminary results from one such trial have been reported [18]. The study was a randomized, double-blind, placebo controlled, 30 day evaluation of the effects of Procera AVH on cognitive function in healthy volunteers. The major cognitive endpoint was the CDR System, a widely used set of automated tests assessing major aspects of cognitive function including attention, vigilance, information processing, working and episodic memory. The preliminary report assessed the overall change after 4 weeks on the various measures from 7 of the 9 tests administered, as well as changes in mood assessed using the Profile of Mood States (POMS). On the basis of this initial analysis, the authors concluded: "Overall, the results suggest that the combination nutraceutical formula, Procera AVH, exerts beneficial effects on both cognition and mood" (Page 18; [18]). The analysis reported here additionally includes data from immediate and delayed word recall tasks which were conducted in the study, as well as a study assessment which was made at 1 week following dosing which was not evaluated in the preliminary report. Further, the CDR System has long had a validated Factor Structure which has been confirmed with principal components analysis [19]. The four major domains which emerge from this analysis are: Power of Attention, the ability to focus attention and process information; Continuity of Attention, the ability to sustain attention, e.g. vigilance; Quality of Memory, the ability to encode, store and subsequently retrieve information held in working memory (both spatial and articulatory) and episodic memory (verbal and non-verbal); and Speed of Memory, the time taken to retrieve information held in working and episodic memory. Since the factor structure was established, it has been used in the vast majority of subsequent publications, including numerous trials in healthy volunteers showing sensitivity to a wide variety of natural products, such energy drinks $[20,21]$, caffeine (e.g. ${ }^{* *}$ ), Guarana (e.g. [22]), Ginkgo biloba (e.g. [23]), Panax ginseng (e.g. [24]), Melissa officinalis (e.g. [25]) and Salvia (e.g. [26]). It has also been used widely in clinical trials of compounds to treat various dementias (e.g. $[21,26,27])$ including a pivotal FDA registration study [28].

\section{Methods}

\section{Design}

Randomized, placebo controlled, parallel group, repeated measures, double-blind study. The study was approved by the Ethical Review Board of Swinburne University.

\section{Participants}

90 healthy male and female participants aged from 22 to 66 years (mean age 48.3 years) were recruited for the study. All gave written informed consent.

\section{Treatment}

On each of the 28 study days, the participants took 3 capsules containing a total of Acetyl-L-Carnitine 1500 $\mathrm{mg}$, Vinpocetine $15 \mathrm{mg}$ and Huperzine A $150 \mu \mathrm{g}$, or 3 placebo capsules which were identical in appearance.

\section{Selection Criteria}

Each participant underwent an individual screening appointment with a registered nurse. Screening comprised a medical history and cognitive assessments. Participants were eligible if they were aged between 22 and 66 years of age; not currently taking prescription drugs affecting the brain or nervous system (e.g., acetylcholinesterase inhibitors, anticholinergics, stimulants, L-dopa, MAO inhibitors, NMDA receptor antagonists, antidepressants), not taking OTC medications affecting the brain (e.g., ephedra based diet pills); not having used any OTC supplements within the past 30 days that have effects on either cognition or mood (e.g. Ginseng, Gingko, Vinpocetine, 5HTP, Tryptophan, St. John's Wort, phosphatidylserine, acetyl-I-carnitine); not active smokers; not taking anti-coagulant drugs (e.g. Warfarin, Heparin, Plavix); anticholinergics or acetylcholinesterase inhibitors; did not have any of the following health conditions: AIDS, HIV, chronic fatigue syndrome, Epstein-Barr, fibromyalgia, lupus, multiple sclerosis, thyroiditis, ulcerative colitis, Crohn's disease, irritable bowel syndrome, dementia including Alzheimer's and Parkinson's disease, Type 1 or 2 diabetes, insomnia or sleep apnoea, narcolepsy; no history of head trauma; no neurological deficits; not pregnant or lactating; not anticipating any planned changes in lifestyle (e.g. exercise regimen) for the duration of the study; and with no known allergies to nuts.

\section{Study Procedure}

\section{Procedure}

During screening each participant completed a general health assessment, which included blood pressure, height and weight, and were then randomly allocated into one of the two treatment groups. They completed a training session on the CDR System which is required in order for the participants to become familiar with the various tests. At the Baseline day they were administered the CDR System followed by the POMS. One and 4 weeks later they again attended the laboratory and again performed the CDR System followed by the POMS.

Participants were requested not to have alcohol or caffeine-containing food or beverages on the three testing days (e.g. coffee, tea, chocolate and energy drinks containing caffeine or guarana). Further on the 
testing days to control for food intake participants were required to eat a light breakfast (e.g. 2 pieces of toast or cereal with juice).

\section{Cognitive testing}

The automated CDR System tests used in this study comprised simple reaction time, choice reaction time, digit vigilance, spatial working memory, articulatory working memory, immediate and delayed word recall, word and picture recognition. These tests have been described previously (e.g. [19]). The tests were administered using a laptop computer and the participants made their responders using a response box containing YES and a NO buttons. For immediate and delayed word recall, the participant wrote the words down on a sheet of paper. The tests were administered by research staff who sat with the participant throughout testing, read preset instructions for each test, then when satisfied the participant understood the test requirements, initiated the test. The data were stored on the computer and also on pen drives. Each test session took around 20 minutes to complete.

\section{Mood testing}

The widely used Profile of Mood States (POMS) is a 65 item self-report questionnaire which has an overall score, named Total Mood Disturbance (TMD). In the preliminary analysis, there were significant reductions in this measure at 4 weeks [18]. This change from baseline at week 4 on the measure was included in the analysis.

\section{Statistical analysis}

Change from baseline data were created by subtracting the scores at weeks 1 and 4 from the baseline scores. These data were analysed for each of the four factors using mixed model repeated measures ANCOVAs conducted with the PROC MIXED procedure from the $\mathrm{SAS}^{\circledR}$ System. Fixed terms were fitted for dosing condition, study week and the interaction between them. Participants were fitted as a random variable. The baseline score was included as a covariate. Degrees of Freedom were adjusted using the Kenward-Roger approximation.
Due to the weight of evidence discussed above indicating that the ingredients in Procera would be expected to improve cognitive function, as is common practice in many research fields [29-31], including regulatory clinical trials [32,33], one-tailed hypothesis testing was employed.

To determine any relationship between changes in cognitive function and mood states, the change from baseline data at week 4 for each CDR System score were correlated with the change in the TMD score from the POMS at this time using the Spearman's rank-order correlation technique.

\section{Results}

CDR System data were available at one or more study days for 73 of the 90 participants who were screened, 46 who had been randomised to active treatment, and 37 who had been randomised to placebo. Of these participants, baseline data plus data from one or both weeks 1 and 4 were available for 44 of the active treated participants and 33 placebo treated participants. The four composite factor scores were calculated using methods described previously $[19,23]$ :

Power of Attention: This is the sum of the reaction time scores from the three attention tests - simple reaction time, choice reaction time and digit vigilance.

Continuity of Attention: This is the sum of the number of correct responses in the choice reaction time and digit vigilance tasks, less the number of false alarm responses in the digit vigilance task.

Quality of Memory: This is the sum of the percentage correct responses from each of the two working memory tasks (numeric and spatial) and the four episodic memory tasks (immediate word recall, delayed word recall, word recognition and picture recognition). It combines a total of 12 accuracy scores from the 6 tasks with a maximum possible score of 600 units.

Speed of Memory: Reaction times for all correct responses are recorded for the two working memory tasks (numeric and spatial) and the two episodic memory recognition tasks (word recognition and picture rec-

Table 1: Results of ANCOVAs for the 4 CDR factor scores.

\begin{tabular}{|l|l|l|l|l|l|}
\hline Measure & $\begin{array}{l}\text { Between Condition } \\
\text { main effect p }\end{array}$ & Condition & LSmeans & SEM & $\begin{array}{l}\text { Change from } \\
\text { baseline p }\end{array}$ \\
\hline Power of Attention (msec) & 0.0872 & Placebo & 11 & 8.13 & 0.1783 \\
\hline Continuity of Attention (nits) & 0.3478 & Procera & -4.1 & 7.4 & 0.5836 \\
\hline Quality of Memory (units) & 0.0294 & Placebo & -0.31 & 0.28 & 0.2701 \\
\hline & & Procera & -0.16 & 0.25 & 0.5132 \\
\hline Speed of Memory (msec) & 0.4793 & Placebo & 8.53 & 5.3 & 0.1095 \\
\hline & & Procera & 22.5 & 5.0 & $<0.0001$ \\
\hline
\end{tabular}


ognition). This score was the sum of the reaction times from these four tasks.

The results of the ANCOVAs are summarized in Table 1 . There were no significant interactions between dose and the weeks of testing for any of the 4 measures, and thus the Table concerns the overall main effects of Procera over weeks 1 and 4 of the study period. The column headed Procera contains the $p$-values for the F-Ratios of the main effect of Procera. The overall mean changes over weeks 1 and 4 for each condition are presented as LSmeans together with the standard errors of the mean (SEM) and 95\% confidence intervals. As the scores are changes from baseline, the $p$-value for the overall change from baseline in placebo or active is presented in the last column for each dosing group.

For Power of Attention, there was a statistical trend for Procera to be superior to placebo, showing an increase in speed over the study compared to a decline with placebo. For Continuity of Attention, there was no significant main effect, although the small decline over the study was greater under placebo than with Procera. On these two measures, neither dosing condition showed a significant change relative to the pre-study baseline levels. For Speed of Memory there was no main effect, but both groups improved over the study to an equivalent extent, this most likely representing a training effect which is sometimes seen on this measure.

Quality of Memory showed a statistically significant main effect of dosing ( $p=0.0294)$, representing a greater improvement over the study in the Procera group compared to the placebo group. Procera also showed a highly significant improvement over the course of the study ( $p<0.0001$ ) compared to the baseline level, while the notably smaller improvement under placebo was not significant. The effect is presented in Figure 1. Thus not only did Procera outperform placebo on Quality

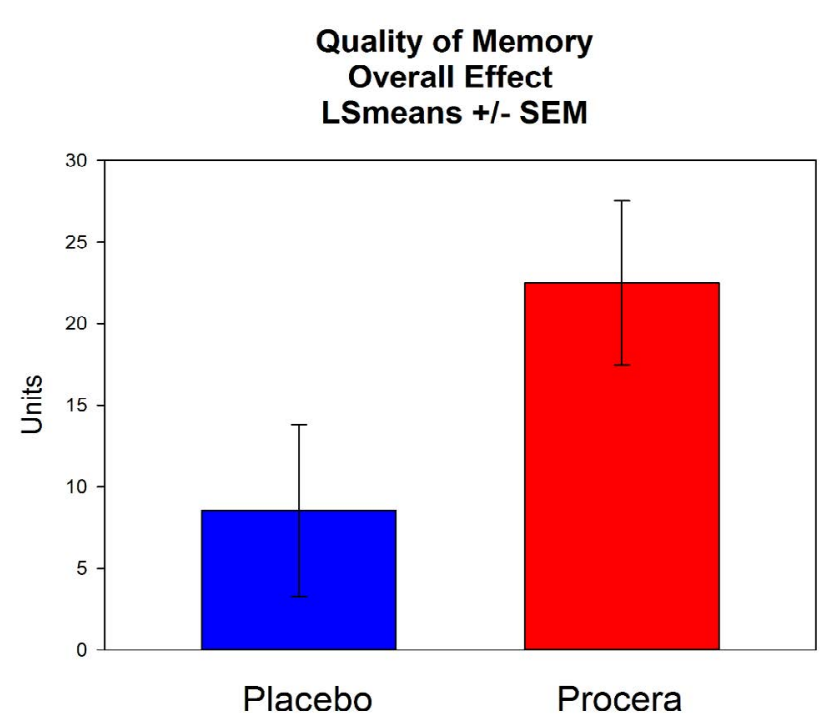

Figure 1: The overall change over the study period for Quality of Memory. of Memory, it also reliably improved the volunteers compared to their pre-study levels.

There were missing data in this study. This can lead to concerns that these missing data have somehow influenced the pattern of results. The most commonly used method for dealing with such situations is a technique named Last Observation Carried Forward (LOCF). This involves replacing missing post-dosing data with scores from previous post-dosing sessions, if available. In the present study, any participant with missing data at week 4 would have any available week 1 data carried forward and substituted for the missing week 4 score. This analysis was performed to determine whether the missing data could have notably influenced the outcome. For Power of Attention, the overall $p$ value from the LOCF analysis was $p=0.0653$, and the mean separation between placebo and active increased slightly from 15.1 to $15.6 \mathrm{msec}$. For Quality of Memory, the overall $p$ value from the LOCF analysis was $p=0.0341$, and the mean separation between placebo and active decreased slightly from 13.97 to 12.41 units. The p-values for the other two factors remained notably non-significant. This additional analysis indicates it was unlikely that the outcome of this study had been notably influenced by the presence of missing data.

The change from pre-dosing scores in the POMS TMD score at 4 weeks were correlated with the changes in Quality of Memory at this time. For the placebo group, there was a non-significant Spearman's Rho correlation in the direction of improved mood being associated with improved Quality of Memory $(r=-0.194 ; p=$ 0.412 ). The correlation in the Procera participants was greater and achieved statistical significance $(r=-0.414$, $p=0.0494)$.

\section{Discussion}

This analysis of the Stough, et al. study using the CDR System Factor scores has identified a statistically significant benefit of Procera AVH on Quality of Memory, a score which combines the accuracy score from the six tests of working and episodic memory. Further, there was a notable and highly significant improvement within the Procera treated participants on this measure of memory compared to their pre-study levels, whereas the smaller improvement under placebo was not statistically reliable. The Speed of Memory score, which reflects the time taken to make decisions in four of the memory tasks, showed an identical change over time in both conditions. Thus the improvements in accuracy on the working memory and episodic recognition memory tasks with Procera cannot be attributed to a change in response strategy, for instance making more accurate responses by taking more time to make them. Overall, this finding for Quality of Memory indicates that it was the ability to store, retain and subsequently retrieve information from short and long term memory stores 
which was improved by the product, as opposed to how quickly the information could be retrieved.

Power of Attention, a measure of focused attention and information processing, showed a statistical trend towards a significant benefit of Procera over placebo. The other composite score from the 3 attention tests, Continuity of Attention, which reflects sustained concentration, did not show statistical support for a benefit of Procera, though the pattern of data was in favour of Procera.

Effect sizes are now widely used to estimate the actual magnitude of benefit of a statistical finding - the larger the effect size the more clinically important the result. One widely used measure of effect size is Cohen's $d$. In the present study, the Cohen's d effect size of the improvement of Procera over placebo on Quality of Memory was 0.38. Rockwood [34] reviewed the effect sizes seen in the clinical trials for the anticholinesterases in Alzheimer's disease. This class of compounds accounts for all but one of the drugs currently licensed to treat this major form of dementia. Rockwood reported a median effect size of 0.28 at the highest effective doses (range 0.01-0.31; [34]). The finding of an effect size of 0.38 in the present study thus indicates that Procera produces improvements which can be considered to be of clinical relevance. Further, the improvement to Power of Attention with Procera over placebo had an effect size of 0.25 , which while not significant compares well to the effect size of 0.28 seen with the anticholinesterases in $A D$.

In the published report on the Procera AVH study [18], the most robust effect of the compound on mood states was a statistically significant and substantial reduction in the Total Mood Disturbance (TMD) scores from the 65 question Profile of Mood States (POMS). The relationships between mood states and the quality of both working and episodic memory have been extensively studied. For example 'sad mood' has been associated with working memory deficits [35], depressed patients being particularly participant to such effects [36]. Conversely, increased emotional arousal is associated with improvements in working and episodic memory [37]. In a study of diet in obese participants, at 8 weeks the POMS TMD dropped substantially and working memory performance improved, both effects being statistically significant [38]. It is thus important that in this study, the improvement to Quality of Memory with Procera correlated significantly with the drop in POMS TMD. This finding, which ties in two independent study outcomes, provides important evidence of the beneficial effects of Procera on both mood and Quality of Memory.

\section{Conclusion}

Overall, this further analysis of the data from the Stough, et al. study has provided statistically reliable ev- idence of the capability of Procera to produce meaningful improvements to a major measure combining the accuracy scores from six working and episodic memory tasks. As the Speed of Memory measure was not differentially affected by Procera and placebo, this indicates that it was the ability to store, retain and subsequently retrieve information from short and long term memory stores which was improved by the product, as opposed to how quickly the information could be retrieved. These improvements on this measure of short and long term memory represent a clinical effect size that exceeds those produced by the current therapies for Alzheimer's disease. Further, the improvements produced by Procera AVH on two major memory systems were also significantly associated with a beneficial effect of the product upon mood disturbance. As Stough, et al. [18] concluded, such positive findings merit follow up in a larger trial.

\section{References}

1. Burlina AP, Sershen H, Debler EA, Lajtha A (1989) Uptake of acetyl-L-carnitine in the brain. Neurochem Res 14: 489493.

2. Lehninger A (1982) Principles of Biochemistry. Worth Publishers, New York.

3. Pande S, Noel H, Murthy M (1986) Characteristics of, function of, and deficiency of carnitine acylcarnitine translocase. In: Borum P, Clinical aspects of human carnitine deficiency. Pergamon Press, New York, 165-180.

4. Piovesan P, Pacifici L, Taglialatela G, Ramacci MT, Angelucci $L$ (1994) Acetyl-Lcarnitine treatment increases choline acetyltransferase activity and NGF levels in the CNS of adult rats following total fimbria-fornix transection. Brain Res 633: 77-82.

5. Taglialatela G, Navarra D, Cruciani R, Ramacci MT, Alema GS, et al. (1994) Acetyl-L-carnitine treatment increases nerve growth factor levels and choline acetyltransferase activity in the central nervous system of aged rats. Exp Gerontol 29: 55-66.

6. Janiri L, Falcone M, Persico A, Tempesta E (1991) Activity of L-carnitine and L-acetylcarnitine on cholinoceptive neocortical neurons of the rat in vivo. J Neural Transmission Gen Sect 86: 135-146.

7. White HL, Scates PW (1990) Acetyl-L-carnitine as a precursor of acetylcholine. Neurochem Res 15: 597-601.

8. Erdo SL, Ning-Sheng C, Wolff JR, Kiss B (1990) Vinpocetin protects against excitotoxic cell death in primary cultures of rat cerebral cortex. Eur J Pharmacol 187: 551-553.

9. Kuzuya $F$ (1985) Effects of vinpocetine on platelet aggregability and erythrocyte deformability. Ther Hung 33: 22-34.

10. Hayakawa M (1992) Effect of vinpocetine on red blood cell deformability in stroke patients. Arzneimittelforschung 42: 425-427.

11. Bönöczk P, Gulyás B, Adam-Vizi V, Nemes A, Kárpáti E, et al. (2000) Role of sodium channel inhibition in neuroprotection: Effect of vinpocetine. Brain Res Bull 53: 245-254.

12. Krieglstein J, Rischke R (1991) Vinpocetine increases the neuroprotective effect of adenosine in vitro. Eur $\mathrm{J}$ Pharmacol 205: 7-10. 
13. Montgomery SA, Thal LJ, Amrein R (2003) Meta-analysis of double blind randomized controlled clinical trials of acetyl-L-carnitine versus placebo in the treatment of mild cognitive impairment and mild Alzheimer's disease. Int Clin Psychopharmacol 18: 61-71.

14. Blaha L, Erzigkeit H, Adamczyk A, Freytag S, Schaltenbrand $R$ (1989) Clinical evidence of the effectiveness of vinpocetine in the treatment of organic psychosyndrome. Human Psychopharmacology 4: 103-111.

15. Fenzl E, Apecechea M, Schaltenbrand R, Friedel R (1986) Long-term study concerning tolerance and efficacy of vinpocetine in elderly patients suffering from a mild to moderate organic psychosyndrome. In: Bes A, Cahn J, Cahn R, Hoyer S, Marc-Vergnes JP, Senile dementias: Early detection. John Libbey Eurotext, London Paris, 580-585.

16. Hindmarch I, Fuchs HH, Erzigkeit H (1991) Efficacy and tolerance of vinpocetine in ambulant patients suffering from mild to moderate organic psychosyndromes. Int Clin Psychopharmacol 6: 31-43.

17. Zangara A (2003) The psychopharmacology of huperzine A: An alkaloid with cognitive enhancing and neuroprotective properties of interest in the treatment of Alzheimer's disease. Pharmacol Biochem Behav 75: 675-686.

18. Stough C, Kure C, Tarasuik J, Lloyd J, Downey LA, et al. (2009) A randomized, double-blind, placebo controlled study examining the effects of a combination nutraceutical formula on cognitive functioning and mood. Journal of the American Nutraceutical Association 12: 12-19.

19. Wesnes KA, Ward T, McGinty A, Petrini O (2000) The memory enhancing effects of a Ginkgo biloba/Panax ginseng combination in healthy middle-aged volunteers. Psychopharmacology (Berl) 152: 353-361.

20. Scholey AB, Kennedy DO (2004) Cognitive and physiological effects of an "energy drink": An evaluation of the whole drink and of glucose, caffeine and herbal flavouring fractions. Psychopharmacology (Berl) 176: 320-330.

21. Wesnes KA, Rinne JO, Hänninen J, Murphy M, Riordan H (2013) Safety and efficacy of ORM-12741 on cognitive and behavioral symptoms in patients with Alzheimer's disease. Journal of Nutrition, Health \& Aging 17: 806.

22. Kennedy DO, Haskell CF, Wesnes KA, Scholey AB (2004) Improved cognitive performance in human volunteers following administration of guarana (Paullinia cupana) extract: Comparison and interaction with Panax ginseng. Pharmacol Biochem Behav 79: 401-411.

23. Kennedy DO, Scholey AB, Wesnes KA (2000) The dose-dependent cognitive effects of acute administration of Ginkgo biloba to healthy young volunteers. Psychopharmacology (Berl) 151: 416-423.

24. Kennedy DO, Scholey AB, Wesnes KA (2001) Dose dependent changes in cognitive performance and mood following acute administration of Ginseng to healthy young volun- teers. Nutr Neurosci 4: 295-310.

25. Kennedy DO, Scholey AB, Tildesley NTJ, Perry EK, Wesnes KA (2002) Modulation of mood and cognitive performance following acute administration of Melissa officinalis (lemon balm). Pharmacol Biochem Behav 72: 953-964.

26. Tildesley NT, Kennedy DO, Perry EK, Ballard CG, Wesnes KA, et al. (2005) Positive modulation of mood and cognitive performance following administration of acute doses of Salvia lavandulaefolia essential oil to healthy young volunteers. Physiol Behav 83: 699-709.

27. Wesnes KA, McKeith IG, Ferrara R, Emre M, Del Ser T, et al. (2002) Effects of rivastigmine on cognitive function in dementia with lewy bodies: A randomised placebo-controlled international study using the cognitive drug research computerised assessment system. Dement Geriatr Cogn Disord 13: 183-192.

28. Emre M, Aarsland D, Albanese A, Byrne EJ, Deuschl G, et al. (2004) Rivastigmine for dementia associated with Parkinson's disease. N Engl J Med 351: 2509-2518.

29. Enkin MW (1994) One and two sided tests of significance. One sided tests should be used more often. BMJ 309: 874.

30. Knottnerus JA, Bouter LM (2001) The ethics of sample size: Two-sided testing and one-sided thinking. J Clin Epidemiol 54: 109-110.

31. Wolterbeek R (1994) One and two sided tests of significance: Statistical hypothesis should be brought into line with clinical hypothesis. BMJ 309: 873-874.

32. Fisher LD (1991) The use of one-sided tests in drug trials: An FDA advisory committee member's perspective. J Biopharm Stat 1: 151-156.

33. Overall JE (1990) Tests of one-sided versus two-sided hypotheses in placebo-controlled clinical trials. Neuropsychopharmacology 3: 233-235.

34. Rockwood K (2004) Size of the treatment effect on cognition of cholinesterase inhibition in Alzheimer's disease. J Neurol Neurosurg Psychiatry 75: 677-685.

35. Chepenik LG, Cornew LA, Farah MJ (2007) The influence of sad mood on cognition. Emotion 7: 802-811.

36. Rogers MA, Kasai K, Koji M, Fukuda R, Iwanami A, et al. (2004) Executive and prefrontal dysfunction in unipolar depression: A review of neuropsychological and imaging evidence. Neurosci Res 50: 1-11.

37. Steidl S, Mohi-uddin S, Anderson AK (2006) Effects of emotional arousal on multiple memory systems: Evidence from declarative and procedural learning. Learn Mem 13: 650-658.

38. Brinkworth GD, Buckley JD, Noakes M, Clifton PM, Wilson CJ (2009) Long-term effects of a very low-carbohydrate diet and a low-fat diet on mood and cognitive function. Arch Intern Med 169: 1873-1880. 